\title{
SOME FACTORS INFLUENCING INFECTIVITY OF THE HUMAN MALARIA PARASITE PLASMODIUM VIVAX (HAEMOSPORIDIA: PLASMODIDAE) TO THE MOSQUITO VECTOR ANOPHELES TESSELLATUS (DIPTERA: CULICIDAE)
}

\author{
M.S. RAMASAMY ${ }^{* *}$, K.A. SRIKRISHNARAJ ${ }^{1 * *}$, S.A.V. MOORTHY ${ }^{2}$, S. \\ JAYAWEERA ${ }^{1}$ and R. RAMASAMY ${ }^{2 * * *}$ \\ ${ }^{1}$ Institute of Fundamental Studies, Kandy. \\ ${ }^{2}$ National Science Foundation, Colombo.
}

(Received : 21 April 2003 ; accepted: 07 June 2004)

\begin{abstract}
Intake of an infective bloodmeal containing Plasmodium vivax gametocytes, by Anopheles tessellatus mosquitoes, leads to the detection of zygotes and ookinetes in the midgut 3-8 h after the bloodmeal. An intact peritrophic matrix, first observed approximately $18 \mathrm{~h}$ after a bloodmeal, is formed irrespective of the body size and age of the mosquito, but only in a proportion of mosquitoes. Unlike in the case of $P$. falciparum where ookinetes develop at about $24 \mathrm{~h}$, the peritrophic matrix would therefore not be a significant barrier to the infectivity of $P$. vivax due to the relatively rapid formation of $P$. vivax ookinetes. The rate of trypsin production was the same in An. tessellatus of different sizes, with the activity peaking about $21 \mathrm{~h}$ post bloodmeal. At this time the majority of $P$. vivax ookinetes would have exited the midgut. A tendency for An. tessellatus of intermediate size to be more susceptible to infection with $P$. vivax than large or small mosquitoes was observed. However this difference was statistically significant in only one of two experiments. The number of oocysts produced, while dependent on gametocytaemia of the blood meal, was not significantly influenced by mosquito size.
\end{abstract}

Key words: Anopheles tessellatus, infectivity, mosquito physiology, peritrophic matrix, Plasmodium vivax.

\section{INTRODUCTION}

Anopheles tessellatus Theobald (Diptera: Culicidae) mosquitoes are natural vectors of the human malaria parasites Plasmodium vivax Grassi and P. falciparum Welch (Haemosporidia: Plasmodidae) in Sri Lanka. ${ }^{1}$ Development of blood-stage gametocytes of malaria parasites and fertilization occurs in the midgut of an Anopheles vector after a blood meal. The resulting zygote loses its spherical shape to become an ellipsoid motile ookinete. Ookinetes reportedly first traverse the peritrophic matrix (PM), a chitinous, lamellar structure that is formed around the blood meal from secretions of the epithelial cells of the midgut. ${ }^{2,3}$ Subsequently, the motile ookinetes cross the midgut epithelium, lodge in the basal lamina' and develop into oocysts in the posterior region of the midgut. There is evidence to

\footnotetext{
We sadly record the untimely demise of Prof. M.S. Ramasamy prior to submission of this manuscript.

** Current address - Vavuniya Campus, University of Jaffna, Vavuniya.

*** Corresponding author, current address - 137/3, Kirula Road, Colombo -05.
} 
suggest that $\mathrm{N}$-acetylglucosamine residues on An. tessellatus midgut epithelium/ PM glycoproteins, chitin or proteoglycans serve as recognition sites for $P$. falciparum and $P$. vivax ookinetes. ${ }^{4,5}$ Sporozoites produced in the oocysts locate to salivary glands, and are inoculated into the host while blood feeding to complete the parasite life cycle.

The intake of a blood meal triggers the synthesis and release of proteolytic enzymes $^{6,7}$ and formation of the PM. Light and electron microscopic studies show that in An. tessellatus, an intact PM is formed approximately $18 \mathrm{~h}$ after a blood meal, beginning in the posterior region of the midgut and generally persisting for up to $48 \mathrm{~h}$ after feeding. ${ }^{8}$ The time taken from fertilization to the formation of the ookinete, the rate of protease production and the rapidity of blood digestion and PM formation are some of the factors that can influence the susceptibility of Anopheles to infection with malaria parasites. Other physiological parameters of the vector mosquito such as body size, age, etc, can also affect the development of the parasite in the vector. Variations in the size of individual mosquitoes are common in nature ${ }^{9}$ and body size reportedly influences the susceptibility of $A n$. gambiae Giles to infection with $P$. falciparum. ${ }^{10,11}$

In contrast to $P$. falciparum, little is known about the rate of development of $P$. vivax ookinetes and the influence of mosquito physiology on their infectivity in vector mosquitoes. This information is important for understanding $P$. vivax transmission and developing improved control measures. The kinetics of ookinete generation, PM formation, protease production, and effect of body size of laboratoryreared An. tessellatus, on the susceptibility to infection by field isolates of $P$. vivax, are reported in this paper.

\section{MATERIALS AND METHODS}

An. tessellatus production: A self-mating colony of An. tessellatus was maintained in the laboratory at $27 \pm 1^{\circ} \mathrm{C}$ and $80 \%$ relative humidity. ${ }^{12}$ The colony was fed on restrained rabbits: the care and use of rabbits were according to WHO guidelines. ${ }^{13}$ Adult An. tessellatus of different body sizes (i.e. L100: large, L200: medium and L400: small) were raised by varying the larval rearing densities at 100, 200 and 400 larvae in 21 of water. ${ }^{14} \mathrm{~L} 100, \mathrm{~L} 200$ and L400 derived mosquitoes had wing lengths of $3.17 \pm 0.02,2.95 \pm 0.02,2.78 \pm 0.03 \mathrm{~mm}$ and body weights of $1.10 \pm 0.04$, $0.90 \pm 0.03$ and $0.60 \pm 0.02 \mathrm{mg}$ respectively.$^{14}$ Mosquitoes produced from L100 larvae were routinely used in experiments unless otherwise stated.

Trypsin activity: The activity of trypsin in the midguts of mosquitoes of different sizes was determined at regular intervals between 15 and $27 \mathrm{~h}$ after a bloodmeal, exactly as previously described. ${ }^{7}$ 
Peritrophic matrix formation: Groups of 3-4 d old L100, L200 and L400 mosquitoes were blood-fed on a rabbit and held in an insectory with access to sugar. At 18, 24 and $30 \mathrm{~h}$ after the intake of the blood meal, the mosquitoes were frozen at $-20^{\circ} \mathrm{C}$. The mosquitoes were dissected using a dissecting microscope, and the midgut epithelium then removed gently to expose the food bolus, to establish the presence or absence of a PM around the posterior end of the bolus exactly as described previously. ${ }^{8}$ For investigating the influence of age on PM formation, 4,7 and $9 \mathrm{~d}$ old L100 mosquitoes were examined in a similar manner for the presence of a PM after a bloodmeal.

Studies on the infectivity of $P$. vivax: The parasitaemia and gametocytaemia of blood used for infection of mosquitoes were determined in Giemsa stained blood films. For determining the kinetics of $P$. vivax ookinete formation, a group of 3-4 $\mathrm{d}$ old L100 mosquitoes from the colony were hand fed on a $P$. vivax infected patient, after informed consent was obtained. Midguts of blood fed mosquitoes were dissected at 1-2 $\mathrm{h}$ intervals from the time of blood feeding until $18 \mathrm{~h}$ afterwards and the epithelium carefully removed. The blood meal was smeared on a glass slide and stained with Giemsa to detect $P$. vivax ookinetes.

For investigating infectivity in mosquitoes of different sizes, parasite infected blood samples were obtained voluntarily, with informed consent, from patients seeking treatment at Government Hospitals at Polgahawela and Rambukkana. These donors had not previously taken drugs for malaria infection. Groups of 3-4 d old L100, L200 and L400 An. tessellatus were then infected with the parasite by a membrane feeding technique described previously. ${ }^{15}$ Blood from the same donor was used in a given experiment. Fully engorged, blood-fed mosquitoes were separated, kept in cages, and dissected $8 \mathrm{~d}$ later for counting oocysts in the midgut. The cumulative mortality of mosquitoes, from the time of the infective blood meal until dissection, was recorded.

Statistical analyses:ANOVA was performed to compare the proportions of mosquitoes of different ages [of a given size, L100] and different sizes [of a given age, 3-4 d old] that developed a PM. Chi-square tests were done to assess the significance of differences between the proportions of mosquitoes infected with $P$. vivax by the membrane feeding technique. The Kruskall-Wallis one-way ANOVA on ranks was used to analyse differences in oocyst numbers between mosquitoes. Tests were carried out using Sigma Stat 2.0 (Jandel Corporation, USA) software.

\section{RESULTS}

\section{Kinetics of the development of P. vivax ookinetes in An. tessellatus}

Microscopic examination of stained smears of the contents of P.vivax infected midguts of An. tessellatus showed the presence of mature zygotes and ookinetes, 3- 
$8 \mathrm{~h}$ after the infective bloodmeal (Figure 1). In contrast, ookinetes were rarely observed in smears made $18 \mathrm{~h}$ after the bloodmeal.
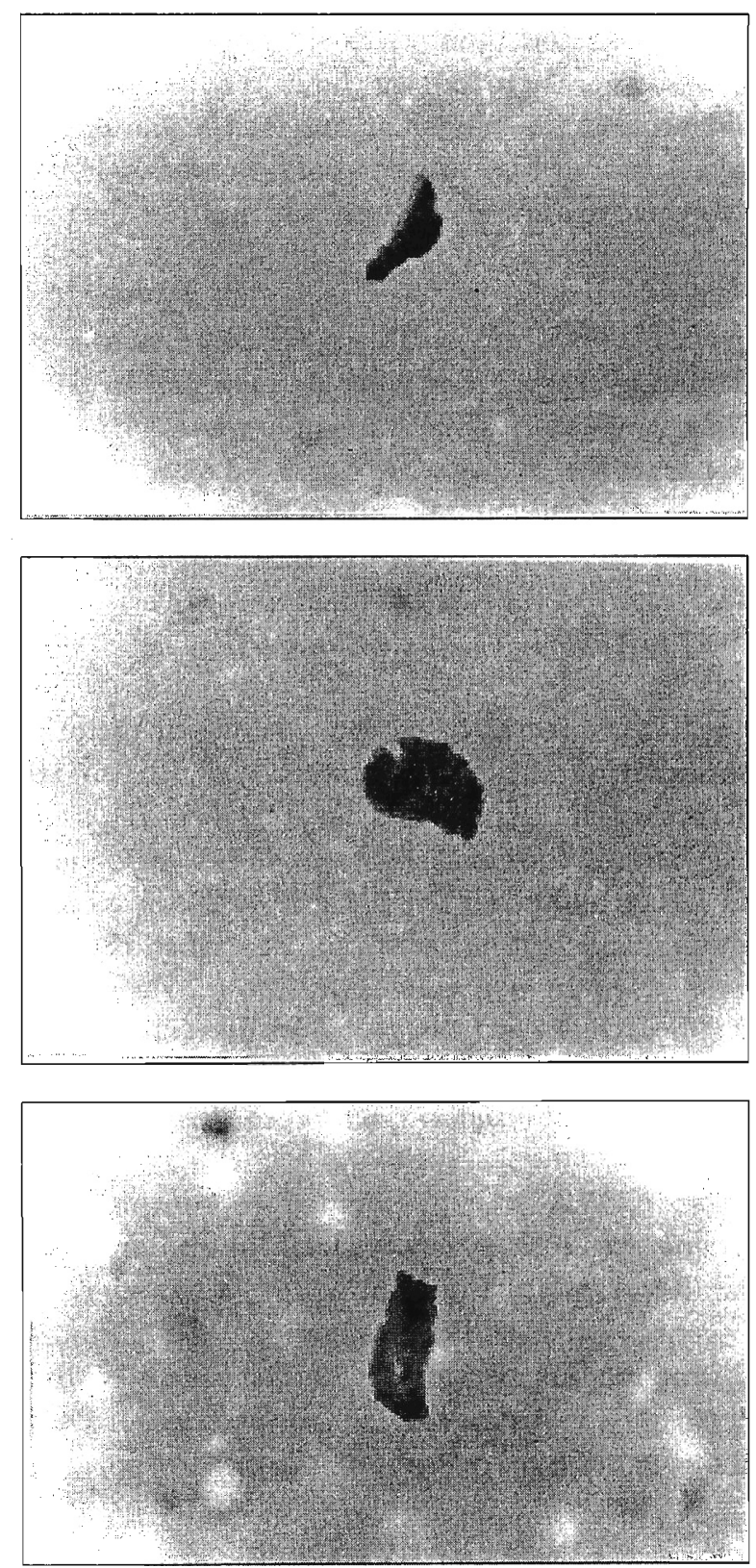

Figure 1a-c: $P$. vivax in smears of the midgut contents of Anopheles tessellatus after the ingestion of a bloodmeal (observed under $\mathbf{x 1 0 0 0}$ magnification). a- ookinete at $3 \mathrm{~h}, \mathrm{~b}$ - zygote at $5 \mathrm{~h}$ and c-ookinete at $8 \mathrm{~h}$, after a bloodmeal. 


\section{Effects of age and size on PM formation in An. tessellatus}

When a PM was present, it was characteristically observed under a microscope as a gelatinous layer extending from the posterior end of the midgut towards the centre of the food bolus. ${ }^{8}$ The PM in An. tessellatus was present in $60-80 \%$ blood fed mosquitoes. The remaining mosquitoes failed to develop a PM. An intact or continuous sleeve of PM was first observed approximately $18 \mathrm{~h}$ after the intake of a bloodmeal.

The proportion of mosquitoes of the L100 size category developing a PM was higher among $4 \mathrm{~d}$ old mosquitoes that took a bloodmeal, than in 7 or $9 \mathrm{~d}$ old mosquitoes (Figure 2). Also an intact PM was observed consistently until $30 \mathrm{~h}$ in a larger number of $4 \mathrm{~d}$ old mosquitoes compared to 7 and $9 \mathrm{~d}$ old ones (Figure 2). However, differences in the proportions of L100 mosquitoes developing a PM were not statistically significant by ANOVA $(p>0.05)$ in mosquitoes of different ages.

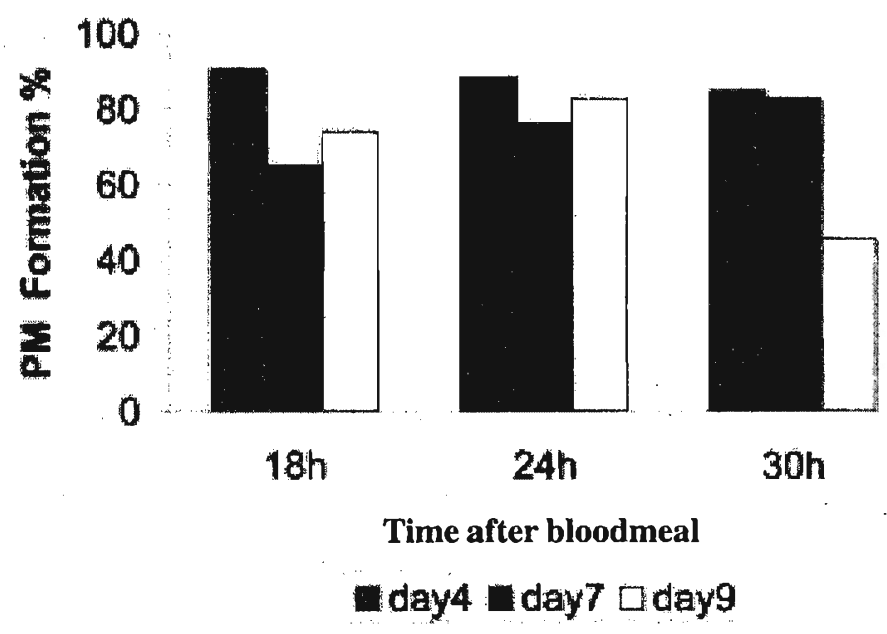

Figure 2: PM formation at different times after a bloodmeal in large sized (L100) An. tessellatus of different ages.

PM formation was also explored in 3-4 d old An. tessellatus of the three experimental sizes. Accordingly, $81 \%(\mathrm{n}=37)$ of $\mathrm{L} 100$ mosquitoes developed a PM after a bloodmeal compared to $68 \% \quad(n=38)$ and $61 \%(n=44)$ of L200 and L400 mosquitoes respectively. More $\mathrm{L} 100$ mosquitoes were observed to have a continuous sleeve of PM at $30 \mathrm{~h}$ after the blood meal compared to L200 and L400 mosquitoes (Figure 3). However, the differences in the proportions of 3-4 $\mathrm{d}$ old mosquitoes of varying sizes developing a PM were not statistically significant by ANOVA $(p>0.05)$. 


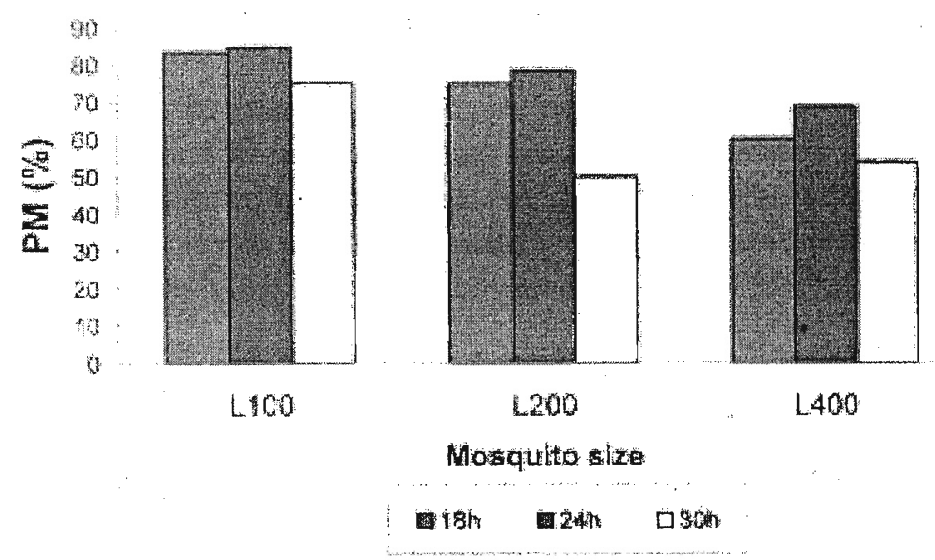

Figure 3: PM formation at different times after a bloodmeal in 3-4 $\mathrm{d}$ old An. tessellatus of different sizes.

\section{Kinetics of trypsin production in An. tessellatus of different sizes}

The development of trypsin activity in the midguts of large (L100), medium (L200) and small (L400)- sized mosquitoes was similar, with maximal enzyme activity occurring about $21 \mathrm{~h}$ after the blood meal in mosquitoes of all three size groups. Detailed data are not shown because they are the same as the kinetics of trypsin production reported previously by us with large (L100) An. tessellatus. ${ }^{7}$

\section{Infectivity of wild P. vivax isolates to An. tessellatus of different sizes}

Three to four day old mosquitoes of all three size groups were readily infected with $P$. vivax (Table 1). In experiment 1, the highest incidence of infection was observed in L200 mosquitoes followed by L400 and L100 mosquitoes. However, in this experiment, the proportions of infected mosquitoes were not significantly related to body size $\left(\chi^{2}=5.66, \mathrm{df}=2, \mathrm{p}>0.05\right)$. In Experiment 2, the proportion of infected mosquitoes was again higher among L200 mosquitoes, than L400 or L100 mosquitoes. In this experiment the proportions of infected mosquitoes were significantly different between the three size categories $\left(\chi^{2}=9.58, \mathrm{df}=2, \mathrm{p}<0.05\right)$.

Oocysts were present mainly in the posterior of the midgut and the numbers of oocysts developed by mosquitoes was dependent on the gametocytaemia of the infective bloodmeal. In Experiment 1, where the gametocytaemia was high, the numbers of oocysts in L100, L200 and L400 mosquitoes analysed by Kruskal-Wallis ANOVA on ranks was not significantly different $(\mathrm{H}=1.55$. $\mathrm{df}=2, \mathrm{p}>0.05)$. This was also the case in Experiment 2 with lower gametocytaemia $(\mathrm{H}=0.52, \mathrm{df}=2, \mathrm{p}>0.05)$. Therefore, the number of $P$. vivax oocysts formed in the mosquito midgut, were not significantly related to mosquito body size. 
Table 1: Effect of body size of An. tessellatus on its susceptibility to infection with $P$. vivax.

\begin{tabular}{cccc}
\hline Mosquito size & $\begin{array}{c}\text { \% Infection* } \\
(\mathrm{n})\end{array}$ & $\begin{array}{c}\text { Oocyst range } \\
{[\text { median }]^{* *}}\end{array}$ & $\begin{array}{c}\text { \% Cumulative } \\
\text { mortality (n)*** }\end{array}$ \\
\hline
\end{tabular}

Experiment 1 gametocytaemia $0.3 \%$

$\begin{array}{llcl}\text { L100 } & 18(56) & 4-226[23]^{\mathrm{a}} & 12.5(64) \\ \mathrm{L} 200 & 39(38) & 3-300[30]^{\mathrm{a}} & 20.8(48) \\ \mathrm{L} 400 & 32(50) & 12-160[65]^{\mathrm{a}} & 26.5(68)\end{array}$

Experiment 2 gametocytaemia $0.06 \%$

\begin{tabular}{llll}
$\mathrm{L} 100$ & $25(24)$ & $01-58[4]^{\mathrm{b}}$ & $69.6(79)$ \\
$\mathrm{L} 200$ & $73(15)$ & $02-72[3]^{\mathrm{b}}$ & $79.7(74)$ \\
$\mathrm{L} 400$ & $33(21)$ & $01-25[5]^{\mathrm{b}}$ & $69.5(69)$ \\
\hline
\end{tabular}

For logistical reasons, (i.e. the quantity of infected blood withdrawn from a patient) the number of mosquitoes used could not be increased. $(n)=$ number of mosquitoes

* In experiment 1 the proportions of infected mosquitoes are not different among the three body sizes $(p>0.05)$. In experiment 2 the proportions of infected mosquitoes are significantly different among the three body sizes $(\mathrm{p}<0.05)$.

:** Medians followed by the same superscripts are not significantly $(\mathrm{p}<0.05)$ different by Kruskall-Wallis one way analysis of variance on ranks.

**** In experiments 1 and 2 the proportions of dead mosquitoes are not significantly different among the three body sizes $(p>0.05)$

The cumulative mortality among infected mosquitoes varied from $12.5-26.5 \%$ in Experiment 1 and from 69.5-79.7\% in Experiment 2. However, mortality was not significantly correlated to body size of the mosquito in either Experiment 1 ( $\chi^{2}=$ 4.05, $\mathrm{df}=2, \mathrm{p}>0.05)$ or in Experiment $2\left(\chi^{2}=2.57, \mathrm{df}=2, \mathrm{p}>0.05\right)$.

Furthermore, the cumulative mortality up to dissection at $8 \mathrm{~d}$, in Experiment 1 where there was higher gametocytaemia in the bloodmeal and consequently higher oocyst numbers on dissection, was lower than in Experiment 2, with lower gametocytaemia and oocyst numbers. This suggests that the intensity of infection with $P$. vivax, as measured by oocyst numbers, influenced mortality less than other aspects of batch to batch variation among mosquitoes.

\section{DISCUSSION}

The kinetics of ookinete formation and penetration in $P$. vivax is different from that of $P$. falciparum, where ookinete development has been observed $24 \mathrm{~h}$ after the vector takes a blood meal. ${ }^{16}$ The maximal ookinete penetration activity occurs at around $22 \mathrm{~h}$ following a bloodmeal in An. atroparvus van Theil infected 
with $P$. berghei $i^{17}$ and between $15-18 \mathrm{~h}$ in An. omori infected with $P$. yeolli nigeriensis. ${ }^{18}$ In Aedes aegypti $(\mathrm{L})$ infected with $P$. gallinaceum, the maximal penetration of the midgut by ookinetes takes place between $30-35 \mathrm{~h}$ after a bloodmeal ${ }^{19}$ and in An. stephensi infected with P. falciparum, maximal ookinete penetration occurs between $32-36 \mathrm{~h}$ after the bloodmeal.$^{20}$ The rate of development of $P$. vivax in the vector midgut, like $P$. yeolli and $P$. berghei, is faster than $P$. falciparum or $P$. gallinaceum. In An. tessellatus, mature oocysts of $P$. vivax are detected 6-7 d after the infective bloodmeal, while those of $P$. falciparum are seen 8-10 d after the infective blood meal. ${ }^{5}$ This may be partly attributed to the ookinetes of P.vivax establishing themselves earlier than those of $P$. falciparum in the basal lamina. Like in other malaria parasites, the oocysts of $P$. vivax establish themselves in the posterior end of the An.tessellatus midgut probably due to the presence of VATPase-over expressing cells in this region. ${ }^{21}$

The PM is formed at varying times in different mosquitoes and it has been suggested that this is one cause for the differential infectivity of Plasmodium for mosquitoes. ${ }^{22}$ In An. stephensi Liston the PM is first seen around $24 \mathrm{~h}^{20}$ and the presence or absence of a PM in An. stephensi did not influence $P$. berghei Vincke infectivity, ${ }^{23}$ although the PM is reportedly a barrier to penetration of $P$. gallinaceum inside the midgut of Ae. aegypti. ${ }^{24} \mathrm{We}$ observe here that ookinetes of $P$. vivax are formed 3-8 $\mathrm{h}$ after the ingestion of the infective blood meal. The intact PM is formed at about18 h, irrespective of the age and size of the An. tessellatus, and this may allow the majority of $P$. vivax ookinetes sufficient time to cross the midgut epithelium and form oocysts. There was no relationship observed between the formation of PM in mosquitoes of different sizes and the relative infectivity of $P$. vivax in the present experiments. Although chitinase synthesised by $P$. falciparum ookinetes helps them penetrate the $\mathrm{PM},{ }^{25}$ there is no evidence for the production of chitinase by the ookinetes of $P$. vivax. These findings are consistent with the proposal that most ookinetes of $P$. vivax cross the midgut before a PM barrier is fully established and do not have to cross the PM before developing into oocyst Because anti-midgut antibodies concomitantly reduce $P$. vivax infection to An. tessellatus ${ }^{15}$ and inhibit PM formation in the posterior midgut, ${ }^{8}$ it was previously postulated ${ }^{26}$ that the PM is not a physical barrier to the establishment of this parasite. Our present findings further support the assertion that the PM is not a significant barrier to the establishment of $P$. vivax infections in An.tessellatus.

Another factor that needs to be considered is whether a bloodmeal containing malaria parasites will influence formation of the PM as a consequence of infection. This possibility was not clarified in the present work but requires further investigation.

The observation that an intact PM is not formed in a proportion 20 An. tessellatus after a bloodmeal warrants further examination. It is argued that the Type I PM, characteristic of fluid-feeding insects, and observed in adult female 
mosquitoes, has evolved primarily to protect against bacteria contaminating the feed. ${ }^{2}$ A PM is absent in some Dipterans that feed on relatively sterile fluids like blood and nectar, ${ }^{2}$ and it may be that it is therefore largely vestigial in adult female mosquitoes. This will be the case if their reproductive ability is not significantly affected by infection with blood-borne parasites. A proportion of blood fed female mosquitoes may therefore not develop a PM, due perhaps to insufficient stimulus, and not show significant ill effects.

When proteolytic enzymes affect the susceptibility of the vector to the malaria parasite, the effects should be evident during the time of zygote to ookinete transition. In An. tessellatus, at 5-6 h after the blood meal, the activity of trypsin in the midgut is low ${ }^{8}$ and therefore, trypsin is unlikely to be deleterious to early $P$. vivax ookinetes. However as the trypsin levels increase in the midgut, later ookinete formation may be significantly affected. Midgut proteases reduce the susceptibility of Anopheles spp, to infection with $P$. falciparum, but the enzymes alone do not affect zygote formation. ${ }^{27}$ In this context, we have previously reported ${ }^{7}$ that the presence of trypsin inhibitors in a bloodmeal delayed the onset of peak trypsin activity by 6-12 h, depending on the inhibitor and its concentration. The trypsin inhibitors increased the infectivity of $P$. vivax but decreased the infectivity of $P$. falciparum to An. tessellatus. ${ }^{7}$ The opposite effects were attributed to delayed trypsin activity reducing damage to zygotes and immature ookinetes of $P$. vivax, while increasing damage to the more slowly developing $P$. falciparum zygote/ookinete or inhibition of the activation of a prochitinase that is required by $P$. falciparum ookinetes. ${ }^{7}$

In laboratory-reared An. dirus, larger females tend to be more frequently infected than smaller females and developed more oocysts when infected with cultured $P$. falciparum although these differences were not statistically significant. ${ }^{28}$ In field caught An. gambiae, larger adults are reported to have fewer sporozoite infections than medium sized mosquitoes. ${ }^{10}$ The proportion of mosquitoes infected was independent of size, but the number of oocysts harboured increased with size of the mosquito. These authors hypothesized that heavier parasite loads in larger mosquitoes would result in greater mosquito mortality in the field. The pattern is interpreted as a combination of high survival rate of large, uninfected mosquitoes and of low survival rate of mosquitoes infected with many oocysts. However, in $A n$. maculatus Theobald naturally infected with $P$. falciparum, $P$. vivax and $P$. malariae, there was no observed correlation between body size and parasite infectivity. ${ }^{30}$ But the same authors reported that females infected with oocysts were significantly larger than those with sporozoites. ${ }^{29}$ Experimental larval rearing densities influenced the adult body size of $A n$. tessellatus and an inverse relationship between wing lengths ranging from $2.78-3.17 \mathrm{~mm}$ and larval rearing densities were observed although the mosquitoes were similar metabolically. ${ }^{14}$ In An. tessellatus infected with $P$. vivax in the laboratory, irrespective of gametocytaemia in the blood meal, there was no consistent relationship between the numbers of oocysts 
formed and body size. The proportion of infected mosquitoes however tended to be greatest with mosquitoes of intermediate size. The cause of this is presently unclear.

Our results demonstrate that mortality of $P$. vivax-infected laboratory-bred An. tessellatus was independent of the body size and, under the experimental conditions, also not affected by the numbers of oocysts. That field caught $A n$. tessellatus did not show increased mortality following infection with the malaria parasite $^{30}$ is consistent with the latter observation. Furthermore, although mortality among field caught An. gambiae was high, the presence of maturing oocysts reportedly did not affect mortality. ${ }^{12}$

\section{Acknowledgement}

This paper is dedicated to the memory of the late Prof. Manthri Ramasamy who wrote the first draft of it. The investigation was supported by the National Science Foundation [RG/95/B/07]. We thank R. Kulasekera and S. Perera for assistance with aspects of the experiments.

\section{References}

1. Mendis C., Gamage-Mendis A.C., de Zoysa A. P. K., Abhayawardana P.A., Carter R., Herath P. R. J. \& Mendis K. (1990). Characteristics of malaria transmission in Kataragama,Sri Lanka: a focus for immunoepidemiological studies. American Journal of Tropical Medicine and Hygiene 42: 293-308.

2 Lehane M.J. (1997). Peritrophic matrix structure and function. Annual Review of Entomolology 42: 525-550.

3 Shen Z. \& Jacobs-Lorena M. (1998). A type 1 peritrophic matrix protein from the malaria vector Anopheles gambiae binds to chitin: cloning, expression and characterization. Journal of Biological Chemistry 273: 17665-17670.

4 Billingsley P.F. \& Sinden R.E. (1997). Determinants of mosquito-malaria specificity. Parasitolology Today 13: 297-301

5 Ramasamy R., Wanniarachchi I.C., Srikrishnaraj K.A. \& Ramasamy M.S. (1997). Mosquito midgut glycoproteins and recognition sites for malaria parasites. Biochimica et Biophysica Acta 1361: 114-122.

6 Briegel H. \& Lea A.O. (1975). Relationship between protein and proteolytic activity in the midgut of mosquitoes. Journal of Insect Physiology 21: 15971604. 
7 Ramasamy M.S., Kulasekera R., Srikrishnaraj K.A. \& Ramasamy R. (1996). Different effects of modulation of mosquito (Diptera: Culicidae) trypsin activity on the infectivity of two human malaria (Hemosporidia: Plasmodidae) parasites. Journal of Medical Entomology 33: 777 - 782.

8 Ramasamy M.S., Raschid L., Srikrishnaraj K.A. \& Ramasamy R. (1996). Antimidgut antibodies inhibit peritrophic membrane formation in the posterior midgut of Anopheles tessellatus Diptera: Culicidae. Journal of Medical Entomology 33: 162-164.

9 Fish D. (1985). An analysis of adult size variation within natural mosquito populations. In: Ecology of Mosquitoes-Proceedings of a Workshop (Eds. L.P. Lounibos, J.R. Rey \& J.H. Frank). Florida Medical Entomology Laboratory. pp 419-429.

10. Lyimo E.O. \& Koella J.C. (1992). Relationship between body size of adult Anopheles gambiae s.l. and infection with the malaria parasite Plasmodium falciparum. Parasitology 104: 233-237.

11 Hogg J.C. \& Hurd H. (1997). The effect of natural Plasmodium falciparum infection on the fecundity and mortality of Anopheles gambiae s.l. in north east Tanzania. Parasitology 114: 325-331.

12 Ramasamy M.S., Srikrishnaraj K.A., Wijekoone S., Jesuthason L.S.B. \& Ramasamy R. (1992). Host immunity to mosquitoes: Effect of antimosquito antibodies on Anopheles tessellatus and Culex quinquefasciatus. Journal of Medical Entomology 29: 934-938.

13 World Health Organisation (1988). Breeding and care of laboratory animals.1\&2. Geneva: WHO.

14 Ramasamy M. S., Srikrishnaraj K. A., Hadjirin N., Perera S. \& Ramasamy R. (2000). Physiological aspects of multiple blood feeding in the malaria vector Anopheles tessellatus. Journal of Insect Physiology 46: 1051-1059.

15 Srikrishnaraj K.A., Ramasamy R. \& Ramasamy M.S. (1995). Antibodies to Anopheles midgut reduce vector competence for Plasmodium vivax malaria. Medical and Veteterinary Entomology 9: 353-357.

16 Meis J., Pool G., Van G.G., Lensen A., Ponnudurai T. \& Meuwissen J. (1989). Plasmodium falciparum ookinetes migrate intracellularly through Anopheles stephensi midgut epithelium. Parasitology Research 76: 13-19. 
17 Sluiters J.F., Visser P.E. \& van der Kaay H.J. (1986). The establishment of Plasmodium berghei in mosquitoes of a refractory and a susceptible line of Anopheles atroparvus. Zeitschrift fur Parasitenkunde 72: 313-322.

18 Syaffruddin R., Aikawa M., Kamimura K. \& Kawamoto F. (1991). Penetration of the mosquito midgut wall by the ookinetes of Plasmodium yeolli nigeriensis. Parasitology Research 77: 230-236.

19 Torri M., Nakamura, K., Seiber, K., Miller, L. \& Aikawa, M. (1992). Penetration of the mosquito (Aedes aegypti) midgut wall by the ookinetes of Plasmodium gallinaceum. Journal of Parasitology 39: 449-454.

20 Meis J.F.G.M. \& Ponnudurai T. (1987). Ultrastructural studies on the interaction of Plasmodium falciparum ookinetes with the midgut epithelium of Anopheles stephansi mosquitoes. Parasitology Research 73: 500-506.

21 Cociancich S.O., Oark S.S., Fidock D.A. \& Shahabuddin M. (1999). Vesicular ATPase- over expressing cells determine the distribution of malaria parasite oocysts on the midguts of mosquitoes. Journal of Biological Chemistry 274: 12650-12655.

22 Ponnudurai T., Billingsley P.F. \& Rudin W. (1988). Differential infectivity of Plasmodium for mosquitoes. Parasitology Today 4: 319-321.

23 Billingsley P. \& Rudin W. (1992). The role of the mosquito peritrophic membrane in bloodmeal digestion and infectivity of Plasmodium species. Journal of Parasitology 78: 430-440.

24 Sieber K., Huber M., Kaslow D., Bank S., Torri M., Aikawa M. \& Miller L. (1991). The peritrophic membrane as a barrier: its penetration by Plasmodium gallinaceum and the effect of a monoclonal antibody to ookinetes. Experimental Parasitology 72: 145-156.

25 Shahabuddin M., Toyoshima T., Aikawa M. \& Kaslow D.A. (1993). Transmission-blocking activity of a chitinase inhibitor and activation of malaria parasite chitinase by mosquito protease. Proceedings of the National Academy of Sciences USA 90: 4266-4270.

26 Ramasamy M.S., Kulasekera R., Wanniarachchi I.C., Srikrishnaraj K.A. \& Ramasamy R. (1997). Interactions of human malaria parasites Plasmodium vivax and $P$. falciparum, with the midgut of Anopheles mosquitoes. Medical and Veterinary Entomology 11: 290-296. 
27 Chege G.M.M., Pumpuni C.B. \& Beier J.C. (1996). Proteolytic enzyme activity and Plasmodium falciparum sporogonic development in three species of Anopheles mosquitoes. Journal of Parasitology 82: 11-18.

28 Kitthawee S., Edman J.D. \& Sattabongkot J. (1990). Evaluation of survival potential and malaria susceptibility among different size classes of laboratoryreared Anopheles dirus. American Journal of Tropical Medicine and Hygiene 43: 328-332.

29 Kittayapong P., Edman J.D., Harrison B.A. \& Delmore D.R. (1992). Female body size, parity and malaria infection of Anopheles maculatus (Diptera: Culicidae) in Pennisular Malaysia. Journal of Medical Entomology 29: 379383.

30 Gamage-Mendis A.C., Rajakaruna J., Weerasinghe S., Mendis K. \& Carter R. (1993). Infectivity of Plasmodium vivax and Plasmodium falciparum to Anopheles tessellatus; relationship between oocyst and sporozoite development. Transactions of the Royal Society for Tropical Medicine and Hygiene 87: 3-6. 